\title{
Zur Periglazialmorphologie von Ura Nippon, der schneereichen Seite Japans
}

An japanischen Beispielen soll die Bedeutung rezenter periglazialer Prozesse in schneereichen Gebirgen der gemäßigten Breiten abgeschätzt werden.

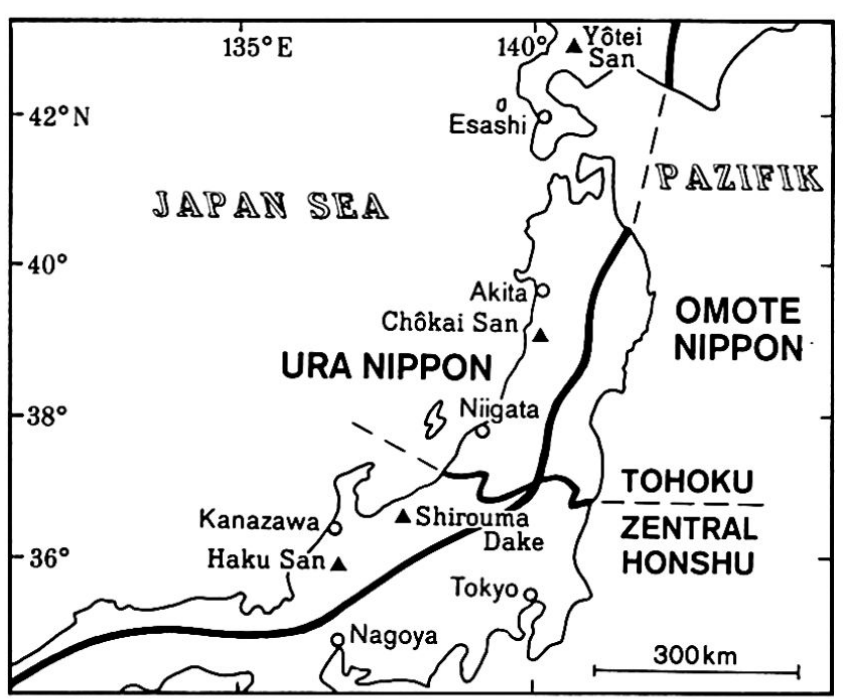

Fig. 1 Geographische Lage von Ura Nippon

\section{Einleitung}

Honshu, die Hauptinsel Japans, erstreckt sich von SW nach NE über $1400 \mathrm{~km}$ und 8 Breitengrade $\left(33^{\circ} 30^{\prime}\right.$ bis $41^{\circ} 30^{\prime} \mathrm{NB}$ ) und umfaßt zwei Klimaprovinzen: Omote Nippon und Ura Nippon.

Omote Nippon, das Gesicht, bzw. die Vorderseite Honshus, ist zum Pazifik hin ausgerichtet. Sie war für die kulturelle und wirtschaftliche Entwicklung Japans von entscheidender Bedeutung, besonders in dem Teil, der südlich von Tôhoku liegt (Tô = Osten, Hoku $=$ Norden, Tôhoku $=$ Nordost-Honshu, Fig. 1). Wie in allen ostasiatischen Monsungebieten besteht in Omote Nippon ein akzentuierter thermischer Jahresgang. Infolge vorherrschender Luftmassenzufuhr von S und SW, d. h. aus tropischen und großenteils marinen Bereichen, sind die Sommer heiß und feucht. Die Winter dagegen, wenn Winde vor allem vom asiatischen Kontinent her, also aus nördlichen Richtungen, wehen und Omote Nippon im Windschatten liegt, sind trocken, schneearm, klar und reich an Ausstrahlungsfrösten.
Ura Nippon ist die «vergessene» Seite von Honshu, wörtlich die Hinterseite. Sie ist der Japan Sea und damit dem asiatischen Kontinent zugewandt. Es mag überraschen, daß man in Japan die dem Festland näher gelegenen Teile als «hinten» empfindet, doch ist die Erklärung einfach: Ura Nippon ist verkehrsgeographisch wegen starker Kammerung der Küstenregion viel schlechter zu erschließen als Omote Nippon. Am meisten behindert aber den wirtschaftlichen Aufschwung ein klimatischer Grund: hier sind die Winter extrem schneereich; Ura Nippon ist das «yuki no kuni», das Schneeland Japans.

\section{Klima von Ura Nippon}

Sowohl Ura als auch Omote Nippon haben Cfa-Klima. Dieses geht gegen $\mathrm{N}$ in Dfa- und in den Bergen in Dfa-, Dfb-, Dfc- und E-Klima über (T. SEKIGUCHI 1965). Ura Nippon nimmt in der Cfa-Klimazone eine Sonderstellung ein, wie aus der Beschreibung des klimatischen Jahresganges (in Anlehnung an M. SCHWIND 1967) hervorgeht.

August. Die höchsten Sommertemperaturen werden zu dieser Zeit erreicht. Die Monatsmittel betragen in Kanazawa $\left(36^{\circ} 33^{\prime} \mathrm{N}\right) 26,0^{\circ} \mathrm{C}$, in Niigata $\left(37^{\circ} 55^{\prime} \mathrm{N}\right)$ $26,1^{\circ} \mathrm{C}$, in Akita $\left(39^{\circ} 43^{\prime} \mathrm{N}\right) 24,4^{\circ} \mathrm{C}$ und in Esashi $\left(41^{\circ} 52^{\prime} \mathrm{N}, \mathrm{SW}-\right.$ Hokkaido) $23,1^{\circ} \mathrm{C}$. Gewitter sind häufig, besonders im südlichen Teil von Ura Nippon.

September. Der Sommermonsun aus S bis SW klingt aus, der Wintermonsun setzt ein. Von $\mathrm{N}$ dringt die Polarfront von Tohoku über Zentral-Honshu nach S vor. Im Kampfgebiet beider Luftmassen kommt es zu heftigen Niederschlägen. Die Welle der Shurin-Regen wandert von $\mathrm{N}$ nach $\mathrm{S}$. Taifune treten bevorzugt in dieser Zeit auf.

Oktober. Taifune kommen nicht mehr vor und die Niederschläge klingen im Zusammenhang mit dem Monsunwechsel rasch ab. Die Auskühlung des Binnenlandes setzt ein.

November, Dezember. Luftmassen aus kontinentalen Bereichen des nordöstlichen Asiens und aus subpolaren marinen Gebieten, die sich immer mehr abkühlen, überstreichen die Japan Sea. Dabei erwärmen sie sich

Dr. L. Ellenberg, Kurfürstendamm 195, 1-Berlin 15. 
über dem vom Tsushima Strom beeinflußten Meer und nehmen Feuchtigkeit auf. In Ura Nippon werden die Winde an den meist schon in Küstennähe steil aufragenden Bergen zum Aufsteigen gezwungen und entladen große Niederschlagsmengen, zunächst noch als Regen, bald aber als Schnee. Der erste Schneefall setzt in den Tieflagen des nördlichen Tôhoku durchschnittlich Ende Oktober ein, im zentralen Ura Nippon gegen Ende November. In den Bergen kommt es zu Schneefällen unmittelbar nach den ersten Frösten, in den Tieflagen sogar meist gleichzeitig mit ihnen. Relativ trocken erreichen Omote Nippon diese winterlichen Luftmassen. Niederschläge sind dort zu dieser Zeit selten.

Januar. Es schneit im Mittel an 20-25 Tagen während des Monats. Während sich die Schneedecke weiter aufbaut, wird die stärkste winterliche Abkühlung erreicht. Die Mittelwerte der Temperatur betragen in Kanazawa $2,2^{\circ} \mathrm{C}$, in Niigata $1,4^{\circ} \mathrm{C}$, Akita $-1,6^{\circ} \mathrm{C}$, Esashi $-1,7^{\circ} \mathrm{C}$. Trotz Insellage und relativ niedrigen Breitengrades sind die thermischen Gegensätze zwischen Sommer und Winter beträchtlich. Die Differenz der Monatsmittel der wärmsten und kältesten Monate beträgt $24-27^{\circ} \mathrm{C}$, ist also um ca. $10^{\circ} \mathrm{C}$ größer als in Mitteleuropa.

Februar, März. Starke Schneefälle halten trotz beginnender Erwärmung an. Im zentralen Ura Nippon erreicht die Schneedecke maximale Werte von $2 \mathrm{~m}$ und mehr (Fig. 2). In den Gebirgen sind Mächtigkeiten von $4 \mathrm{~m}$ keine Seltenheit und am Shirouma Dake in den Japanischen Nordalpen sind an Leeseiten Akkumulationen von über $10 \mathrm{~m}$ Höhe gemessen worden

Der Klimacharakter von Ura Nippon im Vergleich mit anderen Gebieten Japans

\begin{tabular}{l|l|l|l|l}
\hline & $\begin{array}{l}\text { Ura Nippon } \\
\text { zentraler Teil }\end{array}$ & $\begin{array}{l}\text { Ura Nippon } \\
\text { NW-Tôhoku }\end{array}$ & $\begin{array}{l}\text { Omote Nippon } \\
\text { zentraler Teil }\end{array}$ & $\begin{array}{l}\text { Hokkaido } \\
\text { Tokachi Ebene }\end{array}$ \\
\hline $\begin{array}{l}\text { Temperaturen im Januar } \\
\text { Mittelwerte in Tieflagen }\end{array}$ & $\begin{array}{l}\text { mild bis kühl } \\
1-2^{\circ} \mathrm{C}\end{array}$ & $\begin{array}{l}\text { mild bis kühl } \\
-2-1^{\circ} \mathrm{C}\end{array}$ & $\begin{array}{l}\text { mild } \\
2-4^{\circ} \mathrm{C}\end{array}$ & $\begin{array}{l}\text { kalt } \\
-10 \text { bis }-5^{\circ} \mathrm{C}\end{array}$ \\
\hline $\begin{array}{l}\text { Temperaturen im August } \\
\text { Mittelwerte in Tieflagen }\end{array}$ & $\begin{array}{l}\text { heiß } \\
24-26,5^{\circ} \mathrm{C}\end{array}$ & $\begin{array}{l}\text { heiß } \\
23-24,5^{\circ} \mathrm{C}\end{array}$ & $\begin{array}{l}\text { heiß } \\
24,5-26,5^{\circ} \mathrm{C}\end{array}$ & $\begin{array}{l}\text { warm } \\
18-21^{\circ} \mathrm{C}\end{array}$ \\
\hline $\begin{array}{l}\text { Differenz zwischen höchsten } \\
\text { und tiefsten Monatsmitteln }\end{array}$ & $\begin{array}{l}\text { groß } \\
\text { ca. } 24^{\circ} \mathrm{C}\end{array}$ & $\begin{array}{l}\text { groß } \\
\text { ca. } 26^{\circ} \mathrm{C}\end{array}$ & $\begin{array}{l}\text { groß } \\
22-24^{\circ} \mathrm{C}\end{array}$ & $\begin{array}{l}\text { sehr groß } \\
28-31^{\circ} \mathrm{C}\end{array}$ \\
\hline $\begin{array}{l}\text { Niederschläge von November } \\
\text { bis Februar in Tieflagen }\end{array}$ & $\begin{array}{l}\text { sehr groß } \\
\text { bis über } 700 \mathrm{~mm}\end{array}$ & $\begin{array}{l}\text { groß } \\
\text { bis } 600 \mathrm{~mm}\end{array}$ & $\begin{array}{l}\text { klein } \\
\text { meist unter } 300 \mathrm{~mm}\end{array}$ & $\begin{array}{l}\text { klein } \\
\text { unter } 200 \mathrm{~mm}\end{array}$ \\
\hline $\begin{array}{l}\text { Niederschlagssummen im Jahr } \\
\text { in Tieflagen }\end{array}$ & $\begin{array}{l}\text { sehr groß } \\
\text { bis } 2500 \mathrm{~mm}\end{array}$ & $\begin{array}{l}\text { groß } \\
\text { bis } 1800 \mathrm{~mm}\end{array}$ & $\begin{array}{l}\text { groß } \\
\text { bis } 2200 \mathrm{~mm}\end{array}$ & $\begin{array}{l}\text { rel. klein } \\
\text { ca. } 1000 \mathrm{~mm}\end{array}$ \\
\hline $\begin{array}{l}\text { Schneemächtigkeiten im Tief- } \\
\text { land (mittlere Maximalwerte) }\end{array}$ & $\begin{array}{l}\text { sehr groß } \\
\text { bis } 200 \mathrm{~cm} \text { u. mehr }\end{array}$ & $\begin{array}{l}\text { groß } \\
\text { bis über } 120 \mathrm{~cm}\end{array}$ & $\begin{array}{l}\text { sehr klein } \\
0-30 \mathrm{~cm}\end{array}$ & $\begin{array}{l}\text { klein } \\
\text { unter } 100 \mathrm{~cm}\end{array}$ \\
\hline $\begin{array}{l}\text { Mittlere Dauer der geschlosse- } \\
\text { nen Schneedecke in Tieflagen }\end{array}$ & $\begin{array}{l}\text { lange } \\
80-100 \text { Tage }\end{array}$ & $\begin{array}{l}\text { sehr lange } \\
80-160 \text { Tage }\end{array}$ & $\begin{array}{l}\text { sehr kurz } \\
0-15 \text { Tage }\end{array}$ & $\begin{array}{l}\text { sehr lange } \\
\text { ca. } 120 \text { Tage }\end{array}$ \\
\hline $\begin{array}{l}\text { Dauer der schneefreien frost- } \\
\text { wechselaktiven Zeit } \\
\text { im Frühling } \\
\text { im Herbst }\end{array}$ & $\begin{array}{l}\text { kurt fehlend } \\
\text { kurz }\end{array}$ & $\begin{array}{l}\text { i. a. fehlend } \\
\text { sehr kurz }\end{array}$ & $\begin{array}{l}\text { rel. lange } \\
\text { rel. lange }\end{array}$ & $\begin{array}{l}\text { rel. kurz } \\
\text { kurz }\end{array}$ \\
\hline
\end{tabular}


(S. IWATA, Mitt.). Im Winterhalbjahr wird an 100-150 Tagen Niederschlag, und zwar vorwiegend als Schnee, verzeichnet.

April, Mai. Der Wintermonsun klingt aus und die Schneeschmelze beginnt bei sonnenreichem, trockenem Wetter. Doch wird der Schnee auf großen Flächen erst nach der Frostwechselperiode des Frühlings aufgezehrt. Dies gilt gleichermaßen für Hoch- und Tieflagen (Fig. 2).

Juni, Juli. Durch den Sommermonsun wird von SW her die Polarfront gegen Hokkaido hin zurückgedrängt. Analog zu den Shurin-Regen bildet sich eine starke Regenperiode aus, die Bai-u (= Pflaumenregenzeit) genannt wird. Im zentralen Ura Nippon beginnt sie gegen Ende Juni, S-Hokkaido erreicht sie zwischen 10. und 15. Juli. Nun setzt sich die sommerliche Wärme durch. In den Hochregionen Japans treten im Juli und August keine Fröste mehr auf. In den Bergen halten sich immer noch Schneeflecken, von denen einige sogar perennieren.

Den Klimacharakter Ura Nippons im Vergleich mit andern Gebieten Japans zeigt die Tabelle auf S. 140. Wie ganz Japan, ist auch Ura Nippon größtenteils gebirgig. In verschiedener geographischer Breite ragen Einzelvulkane und Gebirgszüge über die Waldgrenze auf, die sich im Relief, im Untergrund und in der Vegetation voneinander unterscheiden. Die Frage, ob in der alpinen Stufe dieser Berge Periglazialprozesse eine nennenswerte Rolle spielen, welche Periglazialformen sie schaffen und in welcher standörtlichen Abhängigkeit diese auftreten, soll im Folgenden nach Beobachtungen an vier Bergen (Fig. l) beantwortet werden. Die Begehungen lagen zwischen Oktober 1972 und Juni 1973 und standen am Shirouma Dake unter der Führung von S. IWATA.

\section{Periglazialformen an Bergen von Ura Nippon}

\section{Haku San}

Haku San, der «Weiße Berg», erreicht 2702 m und liegt auf dem Meridian von Nagoya, also etwa $80 \mathrm{~km}$ im W der Japanischen Alpen. Als Vulkan überragt er ein Bergland aus jurassischen und kretazischen Sedimenten. Er entstand im Quartär durch wenige, große Aus- brüche und war bis in die historische Zeit hinein tätig, zuletzt 1679 (M. SCHWIND 1967). Hornblende-Andesite bedecken als Schutt die meist steilen Hänge und sind großenteils in Tephra eingebettet. Flache Lagen konnten nur an wenigen Stellen entstehen. Verebnungen gibt es stellenweise an der W-Seite des Berges.

Unter Annahme eines Temperaturgradienten von $0,6^{\circ} \mathrm{C} / 100 \mathrm{~m}$ (I. MAEJIMA, unveröff. Manuskript 1973) können folgende Temperaturverhältnisse als wahrscheinlich gelten:

\begin{tabular}{llcc}
\hline Mitteltemperaturen $\left({ }^{\circ} \mathrm{C}\right)$ & Jan. & Aug. & Jahr \\
\hline am Gipfel $(2700 \mathrm{~m})$ & $-15,5$ & 9 & -3 \\
an der Waldgrenze $(2400 \mathrm{~m})$ & -14 & 11 & -1 \\
in 1000 m Höhe & $-5,5$ & 20 & 7 \\
\hline
\end{tabular}

Die Niederschläge überschreiten im Jahresdurchschnitt $3000 \mathrm{~mm}$. Starkregen sind hier wesentlich häufiger als in den weiter nördlich gelegenen Bergen Ura Nippons. Die geschlossene Schneedecke überdauert 120 Tage und wird auch in tiefen Lagen über $2 \mathrm{~m}$ mächtig. Im Mai 1973 fanden sich die untersten Schneeflecken an der W-Abdachung bei $1150 \mathrm{~m}$, im Juni bei $1600 \mathrm{~m}$. Über $1650 \mathrm{~m}$ sind einzelne Nivationsmulden festzustellen; über $2300 \mathrm{~m}$ nehmen solche Mulden alle flacheren Bereiche ein und zeigen dort snowpavements im Schutt.

Oberhalb $1600 \mathrm{~m}$ wird der von Natur aus vorherrschende Laubwald artenärmer und Betula ermanii dominiert. Trockene und flachgründige Standorte werden von Abies mariesii bevorzugt (G. MASAMUNE 1960). Der Unterwuchs dieser montanen Wälder ist dicht und besteht hauptsächlich aus Sasa senanensis. Die Ränder von Schneetälchen werden von Sasa allein besiedelt, Sträucher und Bäume fehlen dort. Die Waldgrenze liegt tiefer als in den schneeärmeren Gebirgen Japans auf gleicher Breite und ist bei ca. $2400 \mathrm{~m}$ anzusetzen. Darüber herrschen Pinus pumila und stellenweise Abies mariesii vor. Wie an allen Bergen Ura Nippons ist die Waldgrenze keine klar sichtbare Linie. Die Baumbestände werden mit zunehmender Höhe artenärmer. Die große Schneehöhe bewirkt Auflösung des Waldes in Streifen oder Gruppen, die auf relativ schneeärmeren Geländerippen oder Kuppen stehen. In den hoch verschneiten Rinnen oder Mulden können sich 
Fig. 2 Schneeverhältnisse. Oben die mittlere Mächtigkeit der Schneedecke im Februar (nach SHAKAI CHIRI); unten die mittlere Dauer der zusammenhängenden Schneedecke (nach NAKAHARA).

Fig. 3 Periglazialformen am Gipfel des Yôtei San.

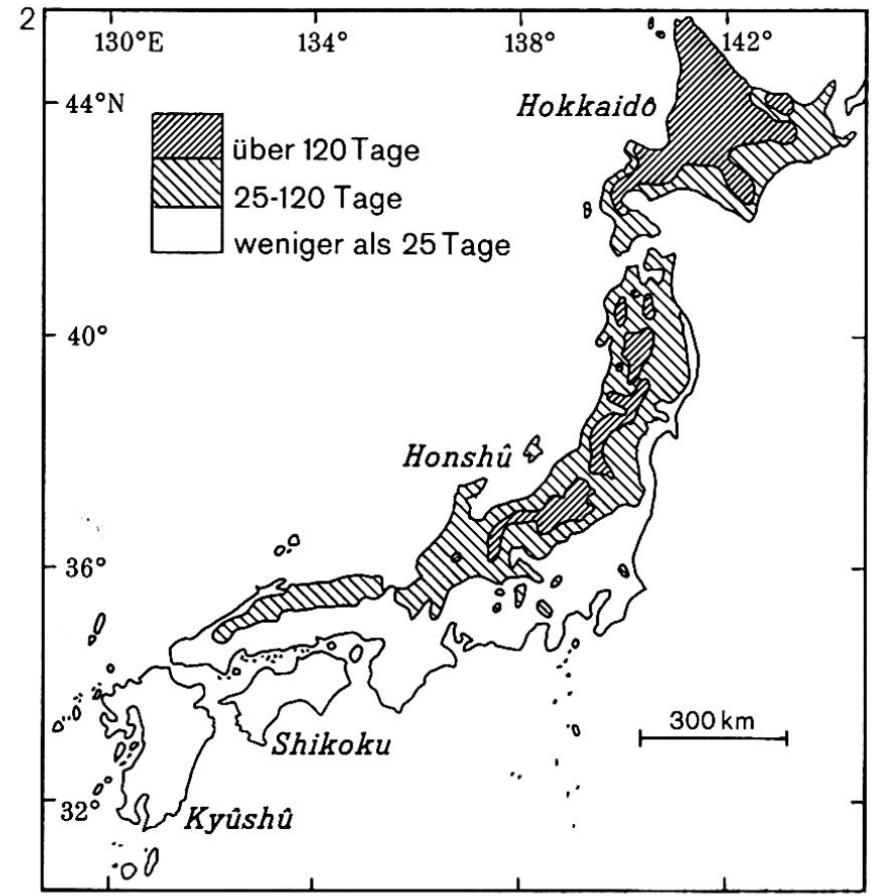

Mittlere Mächtigkeit der Schneedecke im Februar (nach Shakai Chiri)

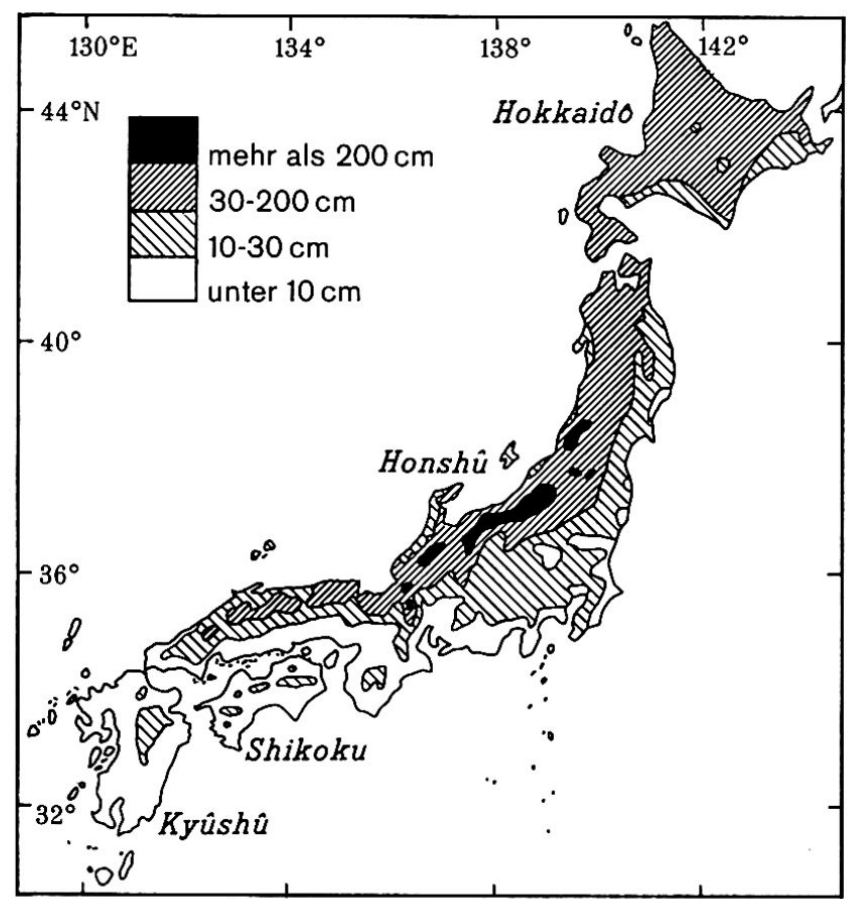

Mittlere Dauer der zusammenhängenden Schneedecke (nach Nakahara)

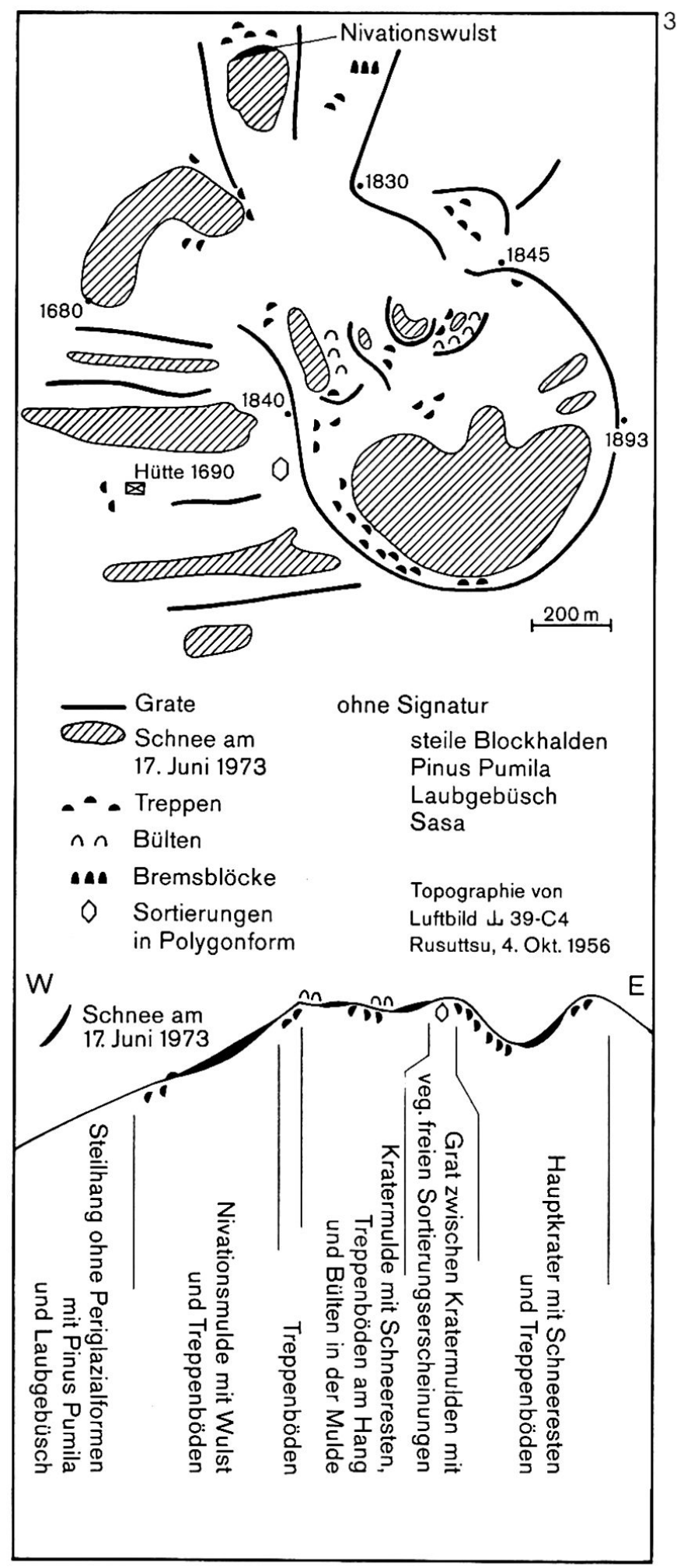


keine Bäume ansiedeln, weil ihr empfindlicher Jungwuchs immer wieder zerstört wird. Hier sind allenfalls niederliegende Sträucher der Last und dem Kriechen des Schnees gewachsen. In den höchsten Lagen und am Rande der Baumgruppen degenerieren die Bäume auch dort, wo sie noch Fuß zu fassen vermochten (H. ELLENBERG, Mitt.).

Periglazialformen sind am Haku San spärlich ausgebildet. Sie kommen in Form kleiner Treppenböden an den Rändern von Schneetälchen oberhalb $1700 \mathrm{~m}$ vereinzelt vor und sind sonst auf winzige Gebiete oberhalb der Waldgrenze beschränkt (Karte von G. MASAMUNE 1960). Im Umkreis einer neuen Berghütte in $2450 \mathrm{~m}$ Höhe sind an der W-Seite zwei Bültenfelder zu finden, die mit zunehmender Hangneigung in scharf konturierte Treppenböden übergehen. Weitere Treppenböden, häufig mit girlandenförmigen Vegetationskanten, sind zwischen Inseln von Pinus pumila an der W-Seite des Berges entwickelt. Im Schutt der fast vegetationsfreien Gipfelregion konnten im Zusammenhang mit Frostrißmustern schlecht entwickelte Sortierungerscheinungen und Ansätze zu Steinstreifen und -ringen gefunden werden. Nirgends sind am Haku San Periglazialformen großflächig ausgebildet.

\section{Shirouma Dake}

Die Japanischen Nordalpen sind das schneereichste Gebiet Japans. In ihrem nördlichen Teil erhebt sich der $2933 \mathrm{~m}$ hohe, auf $36^{\circ} 48^{\prime} \mathrm{N}$ gelegene Shirouma Dake (shiroi $=$ weiß, uma $=$ Pferd, Dake $=$ Berg). Die Hauptmasse dieses Gebirges wird von Quarzporphyren aufgebaut; stellenweise stehen Phyllite, Hornfelse und Serpentine an. Eine für die Japanischen Alpen charakteristische Hang-Asymmetrie ist auch hier deutlich ausgebildet. Von den etwa N-S verlaufenden Hauptgraten dacht sich die W-Seite flach und die ESeite sehr steil ab. Diese Asymmetrie kann z. T. tektonisch erklärt werden (S. KANEKO 1956), ist aber damit nicht hinreichend begründet. Sicher hat die pleistozäne Morphodynamik einen großen Anteil an der Versteilung der E-Seite. Die orographische Schneegrenze lag dort während der Kaltzeiten tiefer als an der windexponierten W-Seite. Spuren von Gletschern, deren tiefste Moränenreste sich bei $1600 \mathrm{~m}$ finden (M. SCHWIND 1967), und Kare sind gänzlich auf die schneereiche E-Seite beschränkt. Sie bewirkten in erster Linie die Versteilung (K. KоBAYASHI 1955, 1956). Nivationsprozesse und starker fluviatiler Abtrag an der Steilseite erhalten im derzeitigen Klima die HangAsymmetrie.

Die Temperaturverhältnisse ähneln denen des Haku San; sie werden in ausgewählten Tagesgängen in verschiedenen Bodentiefen seit 1972 von S. IWATA auf $2450 \mathrm{~m}$ gemessen (unveröff.). Die Niederschläge sind noch reichlicher, besonders im Winterhalbjahr. An der E-Seite treten über der Waldgrenze Schneeakkumulationen bis zu $10 \mathrm{~m}$ und mehr auf, und auch die W-Seite ist tief verschneit. Nur scharfe Grate und windgefegte Hangpartien bleiben ganzjährig aper. Im übrigen Gelände bleibt der Schnee bis in den Juni hinein als geschlossene Decke liegen, an der E-Seite noch wesentlich länger. Perennierende Schneemulden und firngefüllte Rinnen (Abb.1) kommen an der E-Seite vor, wo der Schnee durch Windverfrachtung stark akkumuliert wird (K. HIGUCHI und T. IOZAWA 1971). Man spricht hier von «mannen yuki» (man = Zehntausend, nen $=$ Jahre, yuki $=$ Schnee). Schutt ist fast nirgends locker gelagert; meist sind durch die Schneelast snowpavements ausgebildet. Snow-patch-erosion ist stellenweise für die rezente Morphodynamik von Bedeutung, wie laufende Untersuchungen von S. IWATA zeigen.

Die Schneegrenze läge nach K. KOBAYASHI (1956) bei $4000 \mathrm{~m}$. Obwohl sie nicht erreicht wird, sind Prozesse, die durch Schnee und Schneeschmelze ausgelöst werden, wohl das stärkste morphologische Agens für die Umgestaltung des Reliefs, zumindest in den höheren Regionen.

In den unteren Stufen bildet Laubwald mit Sasa als dichtem Unterwuchs deckende Bestände. Nur einige spät in den Frühling hinein schneegefüllte Rinnen können vom Wald nicht besiedelt werden. Die Waldgrenze, d.h. in diesem Fall das Zurücktreten von schneegedrückten Betula-, Alnus- und Sorbusbeständen und der Übergang zu größeren Pinus pumila-Gebüschen, liegt bei ungefähr $2400 \mathrm{~m}$. Darüber wird das Mosaik der Vegetationsformationen deutlich durch Einflüsse von Schnee, Wind und Boden geprägt. Pinus pumila ist flächig nur an mäßig windexponierten oder sonstigen, nicht zu schneereichen Stellen ausgebildet. Windgeschützte schneereiche Mulden weisen (auch an der W-Seite) eine stark reduzierte Pflanzendecke auf, da dort die Länge der schneefreien Vegetationsperiode für das Aufkommen von höheren Pflanzen 
nicht mehr ausreicht. Ebenso sind stark windgefegte Gratpartien weitgehend ohne Bewuchs. Dieses Gipfelphänomen ist allerdings nur dort deutlich ausgeprägt, wo Gratbereiche vom aus S bis SW her wehenden Sommermonsun bestrichen werden, wo also der Windeinfluß während der Vegetationsperiode bedeutend ist. Sommerlich windgeschützte Hänge können dichten Pinus pumila-Bewuchs bis kurz unterhalb der Kulminationen aufweisen.

Periglazialformen sind fast ausschließlich auf die flache W-Seite beschränkt und liegen dort, von wenigen Ausnahmen abgesehen, über der Waldgrenze. Unmittelbar oberhalb derselben haben sie ihre optimale Verbreitung. Gegen die Gipfel zu wird ihr Auftreten seltener, dies nicht etwa aus klimatischen Gründen, sondern weil das Relief zunehmend steil wird und Fels und Grobschutt als Untergrund vorherrschen.

Ein einziger Fund von Wanderblöcken und Treppenböden wurde östlich des Hauptgrates bei 2570 m ver- zeichnet, und zwar an einem $26^{\circ}$ geneigten Hang in lokaler NE-Exposition. Die Wanderblöcke haben einen Durchmesser bis zu $150 \mathrm{~cm}$; bei den Treppenböden mißt die TF (Terrassenfläche) $70 \times 140 \mathrm{~cm}$, und auf ihr liegen Grobkomponenten bis zu $10 \mathrm{~cm}$ Durchmesser. Die Formen mustern nur eine eng begrenzte Partie des im übrigen dicht mit Pinus pumila bestandenen Hanges.

An der W-Abdachung machen Treppenböden (Abb. 3) den Hauptteil der Periglazialformen aus. Die Vegetationskanten der TH (Terrassenhänge) sind häufig durchbrochen; Schutt wird von oberflächlich abflieBendem Wasser nach Starkniederschlägen durch diese Breschen auf die nächstuntere TF transportiert (L. ELLENBERG 1976). Die TH bleiben im übrigen stabil und sind meist mit dichtem Oxytropo-Kobriesetum bewachsen (T. OHBA 1969).

In $2590 \mathrm{~m}$ Höhe sind einige Wanderblöcke mit Durchmessern von über $100 \mathrm{~cm}$ anzutreffen, die flache Wülste
Schema Shirouma Dake

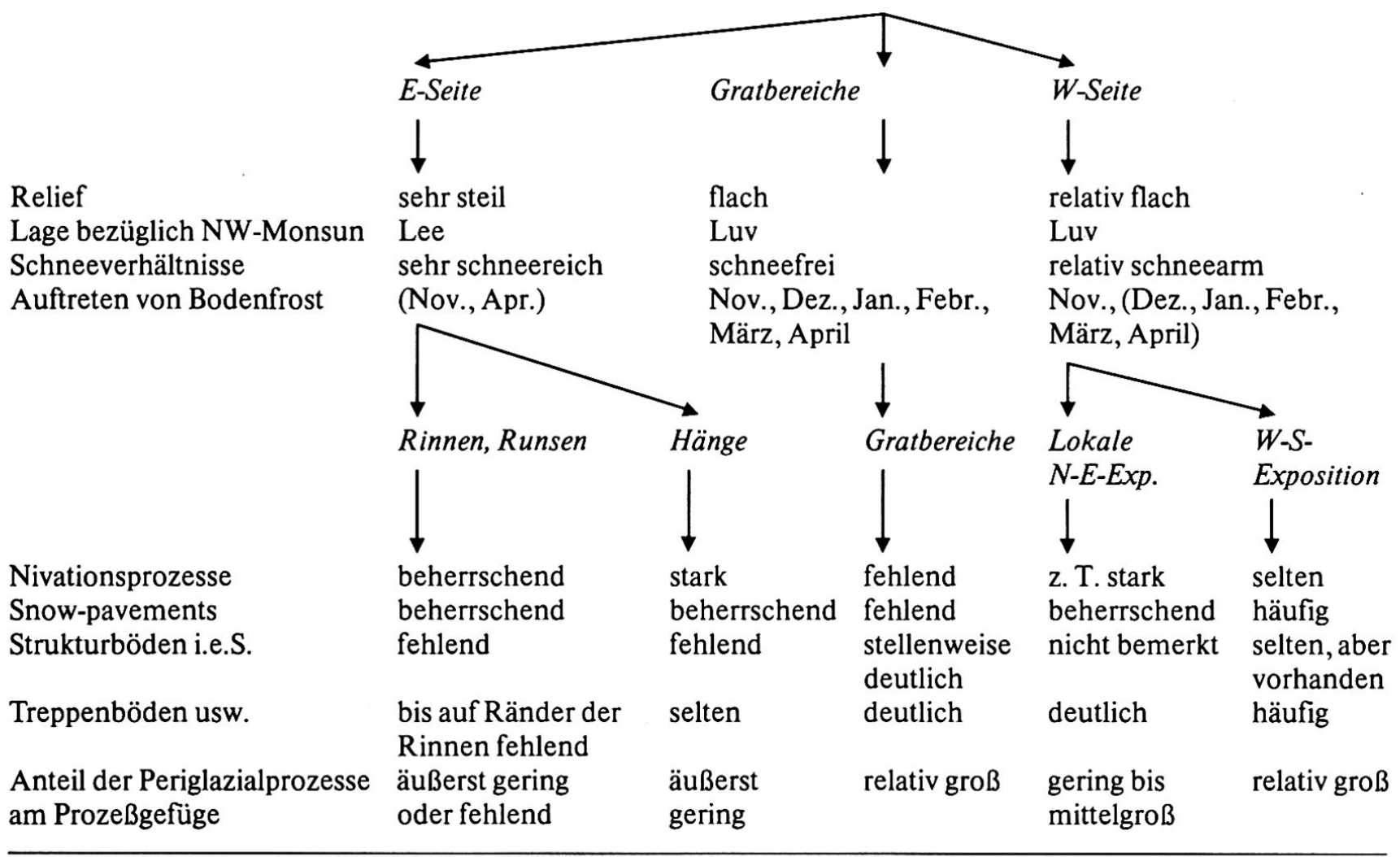


vor sich her schieben und bis $30 \mathrm{~cm}$ tiefe, spärlich bewachsene Auswanderungsnischen hinter sich lassen. Andere Formen der gebundenen Solifluktion fehlen hier ebenso wie auch sonst in Japan, wenn man von Bülten absieht.

Einzelne Blockhalden, die bis $2650 \mathrm{~m}$ hinab reichen, erinnern ihrer Form nach an Blockgletscher. Rezente hangabwärtige Bewegungen sind allerdings nicht auszumachen. In Hokkaido konnten sie dagegen im Gebiet des Daisetsu San beobachtet werden.

Strukturböden im engeren Sinne kommen nur vereinzelt vor, so z. B. als Steinstreifen an einem vegetationsfreien Hang aus Quarzporphyrschutt in lokaler S-Exposition bei $2560 \mathrm{~m}$. Die Streifen der groben Partikel haben hier 15-25 cm Abstand; das Grobmaterial erreicht $8 \mathrm{~cm}$ Durchmesser und die Sortierungstiefe beträgt weniger als $12 \mathrm{~cm}$. Schlecht ausgebildete Steinringe bildeten sich auf einer Verflachung in $2710 \mathrm{~m}$ Höhe und zwar mit Durchmessern von 70 bis $120 \mathrm{~cm}$ und einer Sortierungstiefe von ca. $10 \mathrm{~cm}$.

Typisch für den Shirouma Dake sind schneegedrückte Schutthänge mit Anzeichen von solifluidalen Fließbewegungen. In solchen snow-pavements (Abb. 2,5) mißt S. IWATA seit 1970 die Bewegung des Bodens. Er kommt zu Werten von $2-5 \mathrm{~cm}$ pro Jahr an der Oberfläche, und zwar an einem $18-22^{\circ}$ geneigten Hang in Quarzporphyrschutt, der in lokaler N-Exposition liegt und bis in den Hochsommer hinein schneebedeckt ist. Für frostdynamische Prozesse steht hier nur die kurze Zeitspanne zwischen Beginn herbstlicher Frostwechsel im Boden und starkem Einschneien zur Verfügung. Die Bewegungsraten werden bei zunehmender Bodentiefe rasch kleiner; unterhalb $13 \mathrm{~cm}$ läßt sich kein hangabwärtiger Transport mehr nachweisen.

Bezogen auf den Shirouma Dake sprach M. SCHWIND (1936) von einer Karseite im E und einer Strukturbodenseite im W. Der Begriff «Karseite» ist treffend, der Begriff "Strukturbodenseite» nicht, denn Strukturböden i. e. S. kommen hier nur sehr vereinzelt vor. Allerdings treten andere Periglazialformen - hauptsächlich Treppenböden - häufiger auf. Besser ausgeprägt hingegen sind Formen, deren Entstehung in Zusammenhang mit Schnee oder oberflächlich abfließendem Wasser zu bringen sind. Schnee und fließendes Wasser bestimmen weitgehend die Reliefgestaltung des Shirouma Dake und nicht Periglazialprozesse. Das entsprechende Schema (Shirouma Dake) soll die gemachten Beobachtungen zusammenfassen.

\section{Chôkai San}

Der in Tôhoku bei $39^{\circ} 06^{\prime} \mathrm{N}$ gelegene, $2230 \mathrm{~m}$ hohe Chôkai San erhielt seinen Namen nach einem kleinen See an der W-Seite auf $1600 \mathrm{~m}$ (chôkai = Teich). Er ist ein durch Abtragungsprozesse stark umgestalteter Vulkankegel, der noch in historischer Zeit, zuletzt 1821, aktiv war (M. SCHWIND 1967) und an dem heute noch einige Fumarolen tätig sind. Locker zerfallender Andesit, der meist in Tephra eingebettet ist, herrscht als Grundsubstrat vor. Grobblockige Lagen und Felshänge nehmen die übrigen Teile ein.

Die Temperaturen liegen in gleicher Meereshöhe ca. $2^{\circ} \mathrm{C}$ tiefer als am Shirouma Dake. Die Niederschläge sind etwas geringer (Jahressumme ca. $2500 \mathrm{~mm}$ an der Waldgrenze), doch kann sich im Winter eine Schneedecke aufbauen, deren Mächtigkeit der des Shirouma Dake nur wenig nachsteht.

Die Waldgrenze wird bei ca. $1750 \mathrm{~m}$ erreicht, d.h. 100-200 m tiefer als in gleicher Breitenlage auf der schneearmen Seite Honshus. Durch Schneekriechen bewirkte Verformung an Betula-Stämmen und SasaUnterwuchs setzen schon unterhalb $1000 \mathrm{~m}$ ein. Von $1200 \mathrm{~m}$ Höhe an findet man Birken, deren 10-15 cm dicken Stämme $3 \mathrm{~m}$ dem Hangprofil folgen, bevor sie sich zu ca. $5 \mathrm{~m}$ freier Wuchshöhe erheben. Oberhalb der Waldgrenze sind sie noch häufig in Buschform existenzfähig.

Über der Waldgrenze sind außerhalb von Schutthalden und Felswänden Periglazialformen relativ häufig. Auf einem W-E-verlaufenden breiten Rücken bildeten sich bei ca. $1750 \mathrm{~m}$ Treppenböden. Sie mustern ein Gebiet von 10 ha Größe (Abb. 5). Die TF der Treppen sind ca. $50 \mathrm{~cm}$ breit und können über Zehner von Metern dem Hang folgen. Grobe Fraktionen sind in den obersten $4 \mathrm{~cm}$ des Bodens angereichert und nicht stark gegen die $\mathrm{TH}$ verspült. Breschen in den $\mathrm{TH}$, wie sie für den Shirouma Dake beschrieben wurden, fehlen hier. Eingesprengte Blöcke von bis $60 \mathrm{~cm}$ Durchmesser stören stellenweise das einheitliche Bild. Die Stufenhöhen sind der flachen Hangneigung im Rückenbereich angepaßt und übertreffen $30 \mathrm{~cm}$ nicht. Hier ist es der stete Einfluß des Windes, der eine Schneeakkumulation verhindert und eine dichte und höhere Pflanzendecke nicht aufkommen läßt. Er schafft so günstige Voraussetzungen, $\mathrm{da} ß$ sich die klimatischen Gegebenheiten voll auf die Periglazialformung auswirken können. 
Am Chôkai San haben sich auch unterhalb der Waldgrenze Periglazialformen ausgebildet. Sie sind dort an zwei Standortstypen gebunden: an Flecken mit Fumarolen und an Schneemulden.

An der W-Seite bei $1650 \mathrm{~m}$ verunmöglicht Fumarolentätigkeit den Baum- und Strauchwuchs; die Vegetation ist mattenähnlich. Wanderblöcke sind hier zu finden, deren Bewegung sich nur allmählich vollzieht, wie vollständig bewachsene Auswanderungsnischen verraten. Die Stauchwülste erreichen $1 / 3$ der Höhe der bis $80 \mathrm{~cm}$ hohen Blöcke. Bei Hangneigungen zwischen $5^{\circ}$ und $10^{\circ}$ sind einige Rasenloben ausgebildet, und zwar ähnlich solchen, wie sie H. STINGL (1969) beschreibt. Auf einer größeren Verflachung findet sich ein Bültenfeld mit Aufwölbungshöhen der Buckel bis zu $60 \mathrm{~cm}$. Die vulkanischen Dämpfe sorgen hier für eine relativ lange schneefreie Periode und verhindern ein Aufkommen dichter Vegetation, die den Boden vor Frost abschirmen und durch ihr Wurzelwerk stabilisieren könnte. Hier können sich Periglazialformen nur dank Fumarolentätigkeit bilden.

An anderen Stellen wird die Pflanzendecke nicht durch Fumarolen, sondern durch Schnee reduziert; dies ist der weitaus häufigere Fall. In Schneemulden schmilzt der Schnee erst während des Sommers ab, also nach dem Auftreten der letzten Frühjahrsfröste. Die Vegetationsperiode ist an solchen extrem schneereichen Standorten gegenüber den umliegenden Hangpartien merklich verkürzt. Pinus pumila oder gar Laubgebüsche können nicht aufkommen. Die Mulden haben nur einen schütteren Bewuchs, der den Boden kaum zu stabilisieren vermag. Die kalte Jahreszeit wird in der Regel durch heftige Schneefälle eingeleitet, so daß auch die herbstliche Periode der Frostwechsel und der winterliche Frost ohne großen Effekt auf den Boden bleiben. Treten hingegen vor dem Einschneien in den feuchten Mulden Nachtfröste auf, so üben sie rasch eine Wirkung aus. In nur ein bis zwei frostak-

Schema Chôkai San Gebiet an der W-Seite des Chôkai San über der Waldgrenze

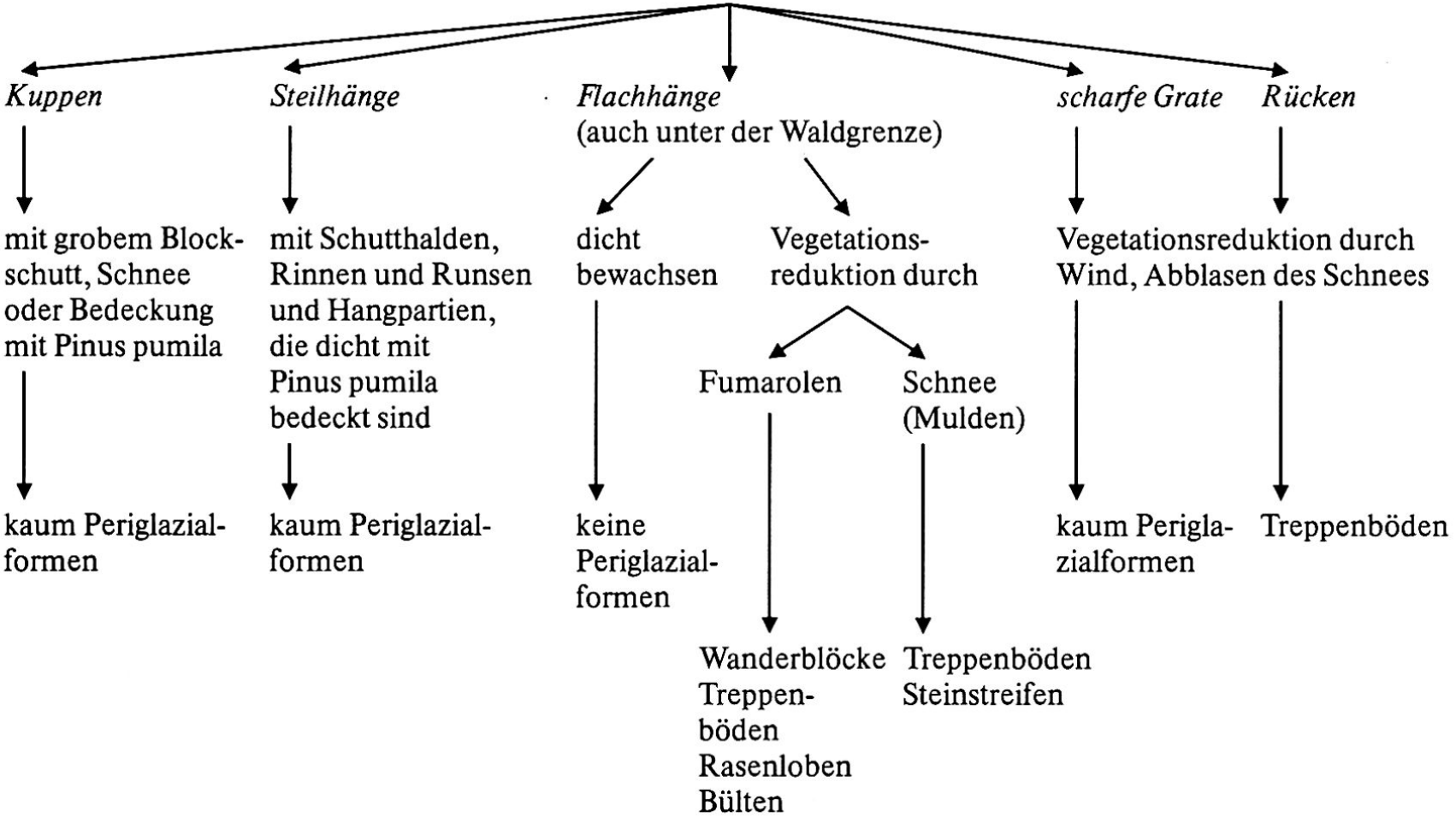


tiven Wochen pro Jahr entstehen hier Treppenböden und an gänzlich vegetationsfreien Stellen Steinstreifen. Bülten können im Muldengrund auftreten. Der Schnee, der die Frostperiode verkürzt und das Eindringen des Frostes in den Boden verhindert, also im allgemeinen der Ausprägung von Periglazialformen entgegen steht, erwirkt hier indirekt über die Reduktion der Vegetation eine Förderung von Periglazialprozessen.

Ähnlich wie für den Shirouma Dake läßt sich für den Chôkai San der skizzierte Sachverhalt schematisch darstellen (siehe Schema Chôkai San).

Unweit des Chôkai San liegt bei $39^{\circ} 55^{\prime} \mathrm{N}$ und $139^{\circ} 55^{\prime} \mathrm{E}$ der Vulkan Kanpĥ San auf der Oga-Halbinsel, der eines der wenigen Beispiele für tiefgelegene Periglazialformen in Ura Nippon bietet. An stark windexponierten, dauernd schneefreien und vegetationslosen Hangpartien sind dort zwischen 250 und 280 m Höhe ü. M.(!) Steinstreifen ausgebildet. Auf dem Gipfel bei $340 \mathrm{~m}$ kommen in Aschen- und Schuttmaterial Polygonnetze mit $10 \mathrm{~cm}$ Maschenweite und $4 \mathrm{~cm}$ Sortierungstiefe vor (Y. SASA 1954).

\section{Yôtei San}

Der Yôtei San liegt zwar im Südzipfel Hokkaidos bei $42^{\circ} 50^{\prime} \mathrm{N}$, gehört aber klimatisch zu Ura Nippon. Der andesitische Stratovulkan ist $1893 \mathrm{~m}$ hoch und hat eine ebenmäßige Glockenform mit einem Durchmesser von $14 \mathrm{~km}$ an der Basis. In seine steilen Hänge sind Runsen nur andeutungsweise eingetieft (Abb. 6). Wegen seiner dem Fuji San ähnelnden Form wird der Berg auch Hokkaido Fuji oder Ezo Fuji genannt (M. SCHWIND 1967).

Folgende Temperaturverhältnisse dürfen angenommen werden:

\begin{tabular}{lllc}
\hline Mitteltemperaturen $\left({ }^{\circ} \mathrm{C}\right)$ & Jan. & Aug. & Jahr \\
\hline in Gipfelbereich $(1850 \mathrm{~m})$ & -14 & 11 & -2 \\
in 1000 m Höhe & -9 & 16 & 3 \\
\hline
\end{tabular}

Die Winter sind hier ähnlich schneereich wie am Chôkai San, die Sommer dagegen trockener; auch gehen Starkniederschläge weniger häufig nieder als in Honshu. Der Einfluß oberflächlich abfließenden Wassers ist weit geringer als z.B. in den Japanischen Alpen. So haben sich bei den Hangrunsen, die kalt- zeitlich periglazial überprägt wurden und dadurch muldenförmige Querschnitte entwickelten, die oberen Partien noch voll erhalten können. Erst unterhalb $1500 \mathrm{~m}$ vollzieht sich ein rascher Übergang zu schärfer und tiefer eingeschnittenen Kerbtälern.

Über der Senke von Kutchan, die landwirtschaftlich genutzt wird, setzt an den steiler werdenden Hängen bei ca. $300 \mathrm{~m}$ Höhe dichter Wald ein. Betula wächst hochstämmig, Farn und Sasa bilden den Unterwuchs. Über $550 \mathrm{~m}$ ist häufig Picea eingestreut, die ihre höchsten Wuchsorte bei $1180 \mathrm{~m}$ erreicht. Im Juni $1973 \mathrm{lag}$ in Rinnen oberhalb $950 \mathrm{~m}$ (W-Seite) noch Schnee. Schneedruckformen sind in allen Rinnen oberhalb $1000 \mathrm{~m}$ deutlich ausgeprägt. Über $1090 \mathrm{~m}$ fanden sich Anzeichen für nächtliche Kammeisbildung, ein Phänomen, das in Ura Nippon auffallend selten vorkommt, ganz im Gegensatz zu Omote Nippon (L. ELLENBERG 1974).

Außer an Treppenböden ist die Abdachung des Yôtei San frei von Periglazialformen. Die Hänge sind hier wohl zu steil und zu dicht bewachsen. Dort, wo im oberen Bereich die Pflanzendecke Barflächen ausspart, können sich wegen der Austrocknung des Oberbodens und damit der Ineffektivierung frostdynamischer Prozesse Periglazialformen nur unvollkommen entwickeln.

Bessere Voraussetzungen sind an der Gipfelkuppe gegeben; sie stellt ein gut ausgeprägtes Periglazialgebiet dar (Fig. 3). Der Kraterrand umschließt einen ca. $100 \mathrm{~m}$ tiefen und $800 \mathrm{~m}$ weiten Hauptkrater und 7 weitere, weniger stark akzentuierte Kraterlöcher. Sie sind teilweise mit Schutthalden ausgekleidet. Schutt bedeckt auch allseitig die Abdachung der Kuppe, bis er sich unterhalb $1700 \mathrm{~m}$ in Pinus pumila-Beständen verfingert. Die Pflanzendecke im Gipfelbereich ist nur schütter, was teilweise durch den Wind und die Schneeakkumulation in Mulden, $z$. T. aber auch anthropogen bedingt sein dürfte. I. FRIEDLÄNDER machte 1909 darauf aufmerksam, daß früher Feuer auf dem Gipfel angelegt wurden, um Regen zu erbitten.

Alle Kraterlöcher waren im Juni 1973, zumindest in nach $\mathrm{N}$ ausgerichteten Lagen, noch schneegefüllt. An den Rändern mit Neigungen zwischen $10^{\circ}$ und $30^{\circ}$ begannen Treppenböden und in den Mulden Bültenfelder auszuapern (Abb.7). Beide Formengruppen ähneln entsprechenden Bildungen im Gebiet des Daisetsu San in Zentral-Hokkaido. Die Bülten sind eng geschart, ihre Scheitel liegen trotz einer Höhe von 
Abb. 1 Shirouma Dake, E-Seite. Firngefüllte Rinne, Blick von 2270 m nach unten. Oktober 1972.

Abb. 2 Shirouma Dake, snow-pavement mit Treppenböden am Rand.
Abb. 3 Shirouma Dake, Treppenböden.

Abb. 4 Chôkai San, Treppenböden auf breitem Rücken, Höhe $1750 \mathrm{~m}$.
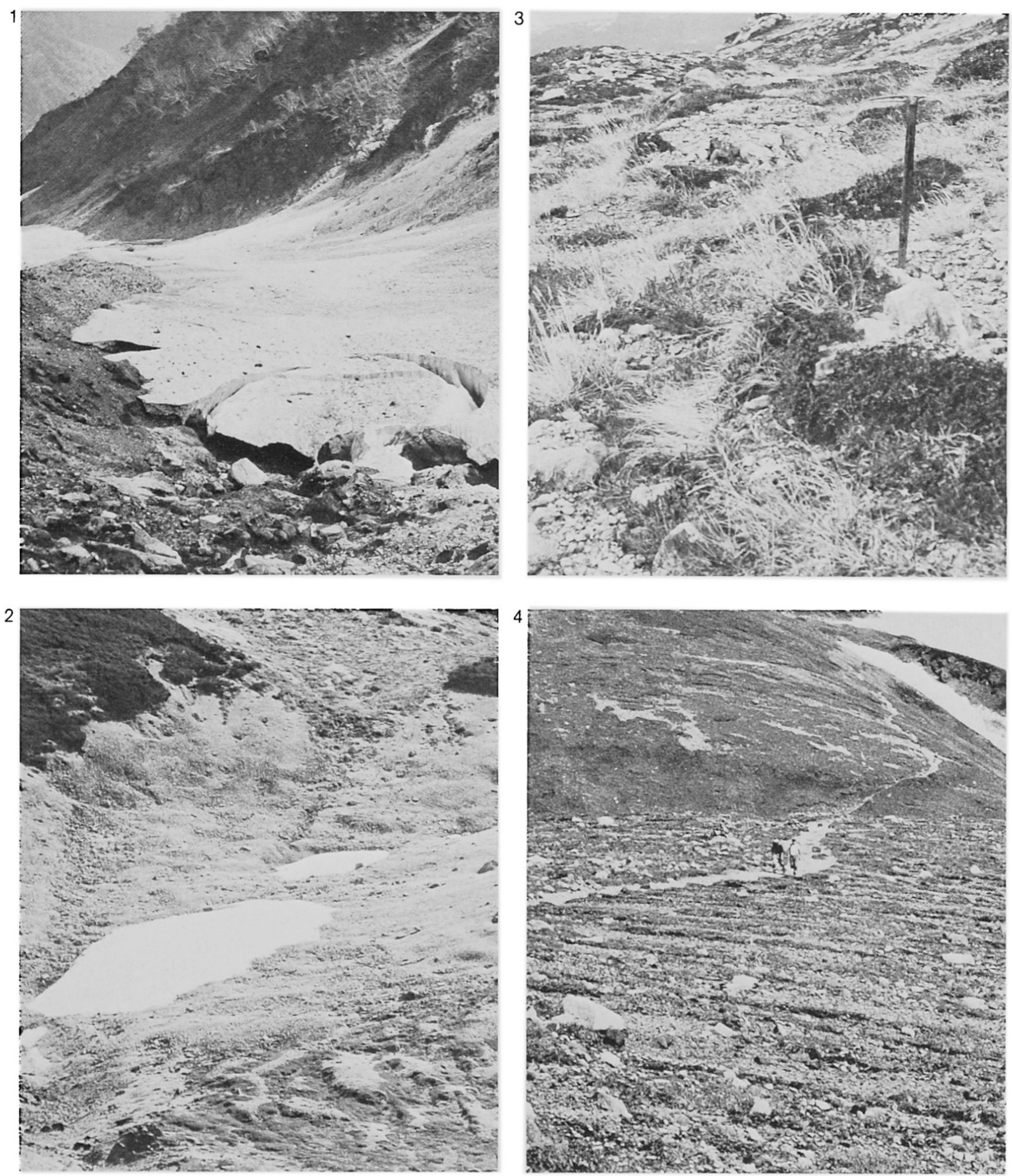
Abb. 5 Shirouma Dake, W-Seite. Im Hintergrund der $2933 \mathrm{~m}$ hohe Hauptgipfel, im Vorder- und Mittelgrund snowpavements mit Anzeichen solifluidalen Fließens.

Abb. 6 Yôtei San von N.

Abb. 7 Yôtei San, Kratermulde mit Bültenfeld, Blickrichtung S.

Abb. 8 Yôtei San, Bremsblock.
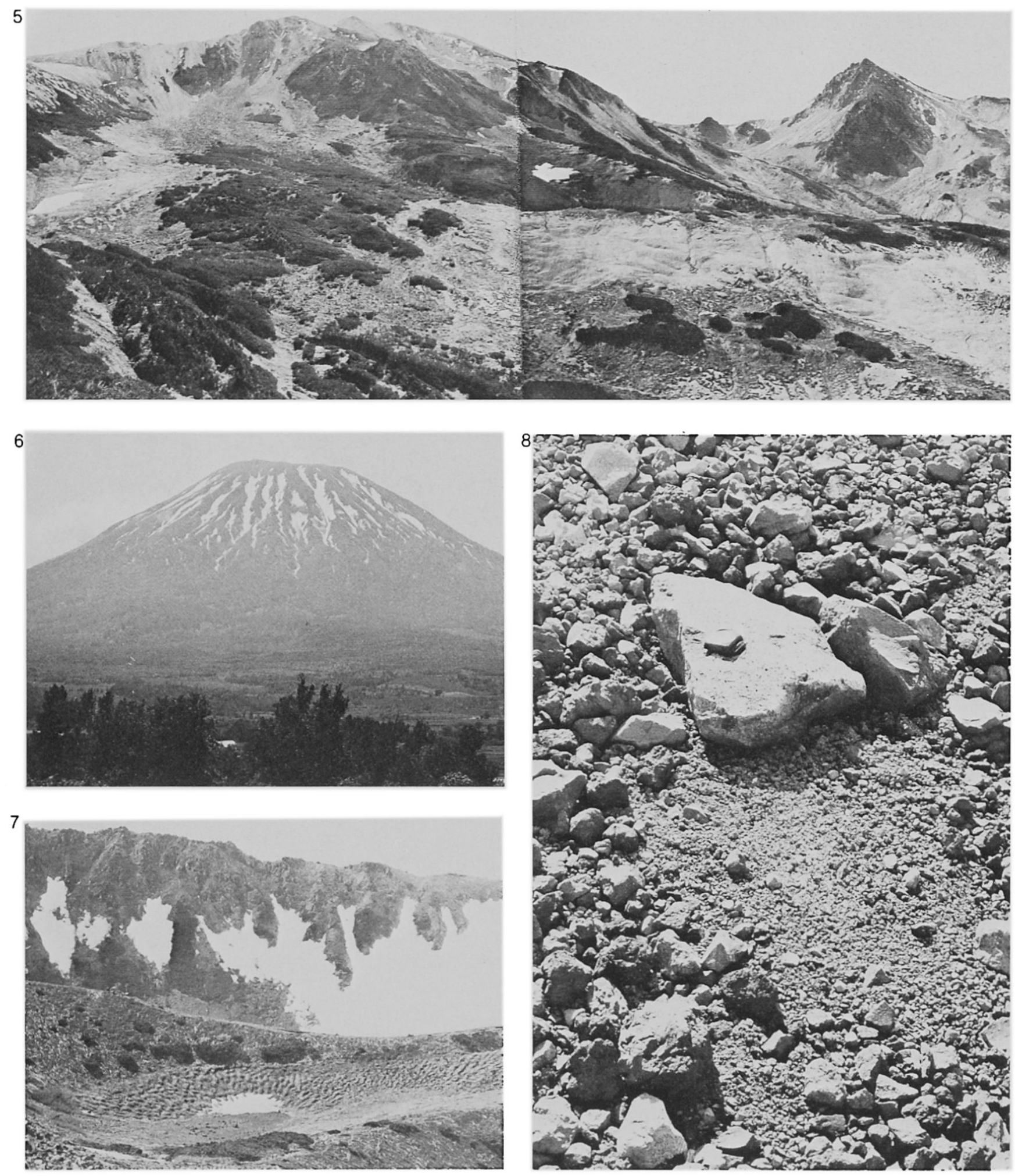
$80 \mathrm{~cm}$ in $150-200 \mathrm{~cm}$ Abstand. Bei leichter Neigung ist das oberflächliche Abfließen von Schmelzwasser an der Ausgestaltung der Rinnenmuster mitbeteiligt. Im N-Teil der Gipfelregion befindet sich eine Schneemulde. In ihrer ursprünglichen Anlage ein Krater, ist sie gegen $\mathrm{W}, \mathrm{S}$ und $\mathrm{E}$ steil begrenzt. Gegen $\mathrm{N}$, wo ein Übergang zur Abdachung des Yôtei San besteht, ist durch Nivationsvorgänge ein Wulst von $150-200 \mathrm{~cm}$ Höhe geschaffen worden. Er liegt bei $1700 \mathrm{~m}$. An seiner N-Stirn sind Treppenböden auffallend gut ausgeprägt, deren steil abgesetzten Vegetationskanten Girlandenform zeigen.

In einigen nach $\mathrm{N}$ abfallenden Schutthalden kommen Bremsblöcke mit Durchmessern bis zu $1 \mathrm{~m}$ vor, die das Material derselben anstauen. Ein anderes Charakteristikum für sie ist der auffallend große Anteil an feinen Schuttfraktionen unterhalb der Blöcke (Abb. 8). Dieser Lee-Effekt verliert sich erst in einer Entfernung, die das drei- bis fünffache des Blockdurchmessers ausmacht.

An einer eng umgrenzten Stelle in $1835 \mathrm{~m}$ Höhe im westlichen Teil des Hauptkraters sind Steinpolygone erkennbar. Sie haben Durchmesser von weniger als $80 \mathrm{~cm}$. Ihre Rahmen lassen sich leicht ausheben; die Sortierung reicht nur wenige $\mathrm{cm}$ tief. An den Hängen gehen sie andeutungsweise in Steinstreifen über.

Der Yôtei San trägt also fast keine Periglazialformen außer in seinem Gipfelbereich.

\section{Periglazialmorphologische Charakterisierung von Ura Nippon}

Die Beobachtungen an den vier Bergen lassen für den rezenten periglazialmorphologischen Befund in den Berggebieten von Ura Nippon folgende Punkte als kennzeichnend erscheinen:

1. Periglazialformen treten nirgends großflächig auf, sie stellen immer Ausnahmeerscheinungen dar. (Das ist in Omote Nippon, wenn auch nicht so kraß, ähnlich, während in Hokkaido im Bereich des Daisetsu San Periglazialformen große Flächen prägen.)

2. Der periglaziale Formenschatz ist klein. Ähnlich wie in den übrigen Berggebieten Japans sind Formen der gebundenen Solifluktion bis auf Bülten und Wanderblöcke fast nirgends zu finden. Formen der gehemmten Solifluktion sind am häufigsten, unter ihnen besonders Treppenböden. Freie Solifluktion kommt vor, ist aber arealmäßig stark eingeschränkt. Die Formen sind nirgends an Permafrost gebunden.

3. An der Gestaltung von Periglazialformen können Druck und Kriechen des Schnees und Spülprozesse, also Formung während des Hochwinters und -sommers, mitbeteiligt sein, wobei sich der Einfluß des oberflächlich abfließenden Wassers wegen abnehmender Niederschlagsintensität gegen $\mathrm{N}$ verringert.

4. An den meisten Stellen wird die periglaziale Formung durch den Schnee verhindert, der die Frostwechselperiode im Boden verkürzt oder aufhebt. Indirekt kann lange Schneebedeckung aber auch förderlich sein, so in Schneemulden, wo das Ausapern so spät einsetzt, daß kaum eine Pflanzendecke aufkommen kann. Hier können in wenigen Herbstwochen mit Frostwechseln Treppenböden gebildet werden.

5. Kammeis, dessen Wirkung im schneearmen Omote Nippon beträchtlich ist, stellt in Ura Nippon kein Agens von großer Wirksamkeit dar.

6. In Andesitschutt, der in Tephra eingebettet ist, kann sich Feuchtigkeit gut halten und für die Ausprägung von Periglazialformen gute Bedingungen schaffen. In locker gelagertem Schutt, der arm an Feinmaterial und windexponiert ist, finden sich keine Periglazialformen.

7. Ein Höhenunterschied zwischen der Solifluktionsgrenze und der Strukturbodengrenze läßt sich in Japan nicht feststellen. Ob Formen der gehemmten oder der freien Solifluktion auftreten, ist allein von der Art und Dichte der Pflanzendecke und nicht vom Klima abhängig.

8. Extrem tiefgelegene Periglazialformen, wie sie im übrigen Japan öfters gefunden werden, sind selten, nicht zuletzt deshalb, weil die Böden in den Tieflagen fast während der ganzen Frostperiode von Schnee bedeckt sind. Fehlt diese Schneedecke und bestehen vom Boden und der Vegetation her keine Hindernisse, so treten sogar Steinstreifen und -polygone in geringer Meereshöhe auf.

9. Im Gelände läßt sich eine Untergrenze des Auftretens von Periglazialformen nirgends kartieren, weil zu wenige Formen auftreten. Die Höhenlage der Untergrenze ist also nicht verläßlich anzugeben. Gehäuft treten die Formen über der Waldgrenze auf. Hierfür sind nicht rein klimatische Gründe verantwortlich zu machen, sondern vor allem die Tatsache, daß die 
Pflanzendecke der periglazialen Formung weniger Hindernisse entgegensetzt. Die Verbreitung der Formen ist in erster Linie durch Vegetation, Schneedecke, Bodenbeschaffenheit und Hangneigung bestimmt, weniger durch besondere Temperaturverhältnisse. Frostwechsel ist zwar Voraussetzung für die Bildung von Periglazialformen aber nicht hinreichend. Mit klimatischen Werten allein kann man nirgends eine Periglazialstufe ausscheiden.

10. Periglazialprozesse sind im morphologischen Prozeßgefüge unbedeutend. Man kann nicht von einer «Periglazialstufe» sprechen, sondern nur von «Periglazialerscheinungen».

Der periglazialmorphologische Befund von Ura Nippon - wenige Formen, die fast ausschließlich über der Waldgrenze liegen - unterscheidet sich deutlich von dem Omote Nippons. Dort sind die Formen etwas häufiger ausgebildet und reichen tief in die Waldstufe hinein.

\section{Literatur}

ELLENBERG, L. (1974) Shimobashira - Kammeis in Japan - Geogr. Helv. 29/1, p. 1-5.

ELlenBerg, L. (1976) Rezente Periglazialerscheinungen auf Cheju-Do, Südkorea - Geogr. Helv., 2, 1976.

FRIEDLÄNDER, I. (1909) Über einige japanische Vulkane - Mitt. Dt. Ges. f. N. - u. V. O.,, Bd. 12, Tl. 1, p. 47-77, Tl. 2, p. 79-154.
KOBAYASHI, K. (1955) An introduction to periglacial or subnival morphology in Japan - Journ. Fac. Lib. Arts and Science, Shinshu University, Nr. 5, p. 23-38.

KobaYASHI, K. (1956) Periglacial morphology in Japan - Rapport de la commission de morphologie périglaciaire de l'UgI, Biuletyn Peryglacjalny, Nr. 4, p. 15-46. MAEJIMA, I. (1973) unveröff. Manuskript über Temperaturgradienten in verschiedenen Gebieten der Erde.

MASAMUNE, G. (1960) Geobotanical Studies of Mt. Hakusan - Ecological studies of Hakusan Quasi-National Park, p. 1-34.

OHBA, T. (1969) Eine pflanzensoziologische Gliederung über die Wüstenpflanzengesellschaften auf alpinen Stufen Japans - Bull. of the Kanagawa Prefecture Museum, Vol. 1, Nr. 2 (japanisch mit deutschen Erklärungen).

SCHWIND, M. (1967) Das Japanische Inselreich, Bd.1, Die Naturlandschaft - Walter de Gruyter \& Co., Berlin. schwind, M. (1936) Die Kasa-Dake-Kette und die Entdeckung des Rundhöckergebietes am Nukedo-Dake The Geographical Review of Japan, 12/4, p. 438-446 (japanisch mit deutscher Zusammenfassung).

SEKIGUCHI, T. (1965) Some problems of climatic classification: a new classification of climates of Japan Japanese Progress in Climatology, p. 1-7.

STINGL, H. (1969) Ein periglazialmorphologisches NordSüd-Profil durch die Ostalpen - Göttinger Geogr. Abhandlungen, 49. 